\title{
Psicologia Escolar e Educacional: um levantamento bibliográfico entre os anos 2008 e 2012
}

\author{
Erico Lopes Pinheiro de Paula \\ Universidade Federal do Triângulo Mineiro - Uberaba - MG \\ Helena de Ornellas Sivieri-Pereira \\ Universidade Federal do Triângulo Mineiro - Uberaba - MG
}

\begin{abstract}
Resumo
Aintensa dispersão epistemológica em torno dos conceitos de Psicologia Escolar e Psicologia Educacional dificulta adoção de critérios consensuais no debate acadêmico. O texto apresentado descreve um levantamento bibliográfico que visa melhor posicionar o foco de investigações ulteriores. Procuramos identificar, em base de dados específica, a ocorrência de termos que se referem à função e atuação dos psicólogos em escolas. A seleção dos trabalhos acadêmicos recentes foi realizada exclusivamente em consulta aos periódicos disponíveis na plataforma SciELO. Os resultados apontam um interesse crescente pela temática e o amadurecimento dos grupos que desenvolvem as pesquisas na área. Para o projeto subsequente, este levantamento indica os mais destacados interlocutores, bem como algumas abordagens produtivas em pesquisa qualitativa. Palavras-chave: Psicologia escolar; psicologia educacional; pesquisa qualitativa.
\end{abstract}

\section{School and Educational Psychology: a literature review between 2008 and 2012}

\begin{abstract}
The intense epistemological dispersion around the concepts of School Psychology and Educational Psychology hinders adoption of agreed criteria in the academic debate. The displayed text describes a literature that aims to better position the focus of further research. We seek to identify, on the basis of specific data, the occurrence of terms that refer to the function and performance of psychologists in schools. The selection of recent academic work has been carried out exclusively in consultation with available journals in the SciELO platform. The results show an increasing interest in the area and the maturing of groups that develop research in the area. For the subsequent project, this survey indicates the most distinguished speakers as well as some productive approaches to qualitative research.
\end{abstract}

Keywords: School psychology; educational psychology; qualitative research.

\section{Psicología Escolar y Educacional: un levantamiento bibliográfico entre los años 2008 y 2012}

\section{Resumen}

La intensa dispersión epistemológica en torno de los conceptos de Psicología Escolar y Psicología Educacional dificulta adopción de criterios consensuales en el debate académico. El texto presentado describe un levantamiento bibliográfico que visa mejor posicionar el foco de investigaciones ulteriores. Se procuró identificar, en base de datos específica, la ocurrencia de términos que se refieren a la función y actuación de los psicólogos en escuelas. La selección de los estudios académicos recientes se realizó exclusivamente en consulta a los periódicos disponibles en la plataforma SciELO. Los resultados apuntan un interés creciente por la temática y la maduración de los grupos que desarrollan las investigaciones en el área. Para el proyecto subsecuente, este levantamiento indica los más destacados interlocutores, así como algunos abordajes productivos en investigaciones cualitativas.

Palabras clave: Psicología escolar; psicología educacional; pesquisa cualitativa. 


\section{Introdução}

Este texto descreve uma pesquisa que integra um projeto mais abrangente denominado "Educação e Psicologia: representações sociais de professores na rede pública”. Classificamos a etapa aqui descrita entre as pesquisas que segundo Ferreira (2002) apresentam caráter inventariante e descritivo sobre determinado tema. O estudo foi concluído no primeiro semestre de 2014.

No projeto justificamos que a presença do psicólogo na escola produz condições específicas para a realização do trabalho educacional, tanto para a atuação do psicólogo quanto para os outros profissionais efetivos no quadro do magistério. Reportamos o ingresso do psicólogo na escola tomando por base a discussão em torno do Projeto de Lei Complementar $n^{\circ} 3688$, apresentado na Câmara dos Deputados no ano 2000 pelo deputado José Carlos Elias (PTB/ ES), que prevê a inserção de psicólogos e assistentes sociais no quadro de funcionários de todas as escolas públicas de educação básica no Brasil (Brasil, 2010).

Nas leituras preliminares encontramos o debate sobre a intensa dispersão epistemológica em torno dos conceitos de Psicologia Escolar, Psicologia Educacional e Psicologia da Educação. Entendemos aqui a dispersão epistemológica de maneira semelhante àquela proposta por Martino (2013), quando analisa a (des)organização curricular da disciplina Teoria da Comunicação, ou a exemplo do "inconveniente" apontado por Mazzotti (2006) nas Ciências do Homem. Dessa forma, procuramos recortar o universo com o qual dialogamos, estabelecendo-o como: conjunto dos artigos veiculados em periódicos científicos, em período recente, que tratam de Psicologia e Educação Escolar, com ênfase em Psicologia Escolar e Educacional.

O objetivo geral desta investigação foi o de identificar e interpretar os resultados de estudos sobre o papel do psicólogo escolar e educacional na atualidade. Os objetivos específicos resumem-se a: definir o conjunto de periódicos que atendiam aos critérios de inclusão; selecionar os artigos com aplicação de descritores; realizar leitura analítica e ilustrar os resultados por meio de tabelas e gráficos. Com a conclusão esperamos colaborar para a entrada do psicólogo no sistema público de ensino, disponibilizando referências e instrumentos adequados para o debate público e para a participação coletiva nas decisões sobre a educação formal.

\section{Método}

A escolha dos periódicos na base de dados levou em conta os seguintes critérios de inclusão: (a) vínculo com Psicologia e Educação, definidas pelas áreas de avaliação do Qualis/CAPES; (b) qualidade e tradição, periódico classificado como A1, A2, B1 e B2 (tanto na área Psicologia quanto na Educação); e (c) atualidade, que reúne artigos produzidos entre 2008 e 2012. Os critérios de exclusão utilizados foram: (a) apenas periódicos editados no Brasil com produção em língua portuguesa; e (b) apenas os disponíveis integralmente na plataforma do Programa Scientific Eletronic Library Online (SciELO). Quando a revista estava hospedada em sítio próprio, ou quando só havia versão impressa da mesma, a publicação foi excluída dessa revisão.

O levantamento teve início com a escolha dos periódicos a partir da classificação disponível no WebQualis ${ }^{1}$. No primeiro momento as revistas foram selecionadas pela classificação na área de Psicologia, considerando-se apenas os estratos $A 1, A 2, B 1$ e $B 2$. Em tabela à parte foram registrados nomes e outros dados - ISSN e links de acesso na internet - porém, parte dos registros foi omitida por motivo de espaço nesta edição. Oferecemos um exemplo restrito na Tabela 1:

Voltamos ao sítio da Coordenação de Aperfeiçoamento de Pessoal de Nível Superior (CAPES) para consultar cada um dos ISSN na busca individual. Verificamos neste procedimento a classificação quanto à área de Educação - e excluímos os artigos não classificados nesta área ou contidos nos estratos B3 e posteriores. Após a sondagem, restaram 74 publicações mantidas em versão online integral na plataforma SciELO². Com a ferramenta de busca disponível no próprio repositório, selecionamos os textos a partir do título, do resumo e do corpo do texto. Foram considerados aceitos aqueles trabalhos nos quais aparecessem um dos seguintes termos: "Psicologia Escolar", "Psicologia Educacional", "Psicólogo Escolar" ou "Psicólogo Educacional". Por fim, os artigos foram copiados em pasta de trabalho.

Em seguida consideramos revisões preliminares que nos permitiram classificar os artigos em três categorias de análise, relativas aos objetivos e/ou os resultados relatados nas respectivas pesquisas:

1 http://qualis.capes.gov.br/webqualis/principal.seam

2 http://www.scielo.org/php/index.php

Tabela 1. ISSN, título e endereço eletrônico dos periódicos selecionados no estrato A1

\begin{tabular}{l|l|l}
\hline \multicolumn{1}{c}{ ISSN } & \multicolumn{1}{c}{ Periódico } & \multicolumn{1}{c}{ link } \\
\hline $\mathbf{0 1 0 3 -}$ & Paidéia (USP. Ribeirão Preto. & http://www.scielo.br/scielo.php?script=sci_serial\&pid=0103-863X \\
$\mathbf{8 6 3 X}$ & Impresso) & \\
\hline $\mathbf{0 1 0 2 -}$ & Psicologia: Reflexão e Crítica & http://scielo.br/scielo.php?script=sci_serial\&pid=0102-7972\&Ing=pt\&nrm=isso \\
$\mathbf{7 9 7 2}$ & (UFRGS. Impresso) & \\
\hline $\mathbf{0 1 0 2 -}$ & Psicologia: Teoria e Pesquisa & http://scielo.br/scielo.php?script=sci_serial\&Ing=pt\&pid=0102-3772\&nrm=iso \\
$\mathbf{3 7 7 2}$ & (UnB. Impresso) & \\
\hline
\end{tabular}

Fonte: Os autores 
- "a" - qualitativo específico: artigo que relata pesquisa qualitativa diretamente relacionada com o projeto mais amplo - pelo contexto de aquisição e análise dos dados (abordagens, sujeitos), pela escolha dos instrumentos (questionários, entrevistas, grupos focais), pelos sujeitos envolvidos (especialmente professores e psicólogos em atuação na escola) e/ou pelos objetivos almejados (interpretar manifestações discursivas e concepções);

- "b" - teórico-instrumental: artigo que apresenta trabalhos teóricos, resultado de estudos bibliográficos, revisões históricas ou discussões epistemológicas - legislação e literatura acadêmica - sobre a trajetória da Psicologia Escolar como especialidade científica e/ou da formação do profissional da psicologia que atua em escolas (em contextos brasileiro, regional ou local);

- "c" - diversos: artigo que não se enquadra nas categorias anteriores, porque abarca temas específicos da psicologia em geral (educação inclusiva, psicologia sanitária, saúde pública, comportamento, bullying, entre outros) e/ ou realiza estudo de campo com sujeitos ou contextos distantes dos propósitos da pesquisa ulterior.

Mediante os procedimentos descritos foi composto o "corpus definitivo" da pesquisa, contendo o total de 58 artigos. Também foi necessário fazer a leitura do artigo completo para tornar mais claros os aspectos metodológicos ou procedimentais das pesquisas. Diante disso, foram identificados os caracteres que permitiram classificar os artigos em uma das seguintes categorias: "a" - qualitativo específico;"b" - teórico-instrumental;"c" - diversos.

As informações recolhidas na leitura inicial foram sistematizadas em tabelas e serviram para a identificação das ocorrências. Finalmente, foram produzidos os gráficos ilustrativos que constam na seção final deste artigo. A última etapa do levantamento consistiu em discussão sumária sobre os resultados encontrados em alguns dos textos mais relevantes.

\section{Resultados}

O grupo de artigos a ser analisado contabilizou 58 trabalhos veiculados nos quatro estratos escolhidos - distribuídos como na demonstração da Figura 1.

Encontramos os três artigos do estrato A1 distribuídos na frequência: um trabalho em 2008, 2010 e 2011. Identificamos também a ocorrência dos 18 artigos encontrados no estrato A2: um artigo em 2008 e outro em 2009, cinco artigos em 2011 e em 2012, e seis trabalhos publicados em 2010. No estrato B1, registramos o total de 32 trabalhos até 2012: dois artigos em 2008, quatro em 2009, oito em 2010, nove em 2011 e 2012. As ocorrências no estrato B2 perfazem um total de cinco artigos: dois em 2011, dois em 2012 e uma publicação em 2010.Esse aspecto indica a tendência de crescimento quantitativo e qualitativo das pesquisas nesse campo de aplicação.
Figura 1. Corpus definitivo por estrato e por ano.

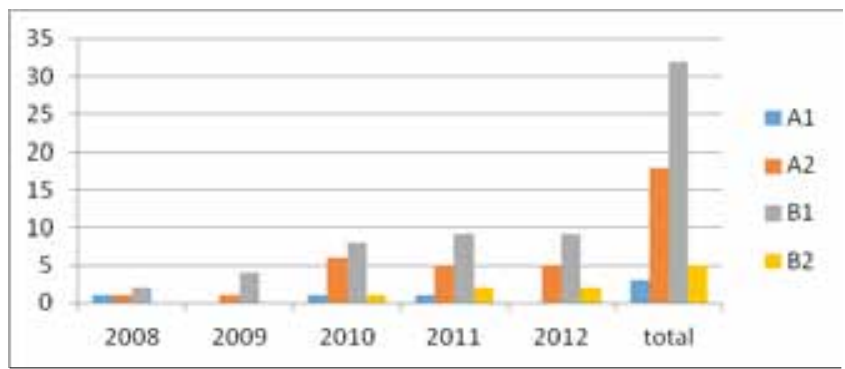

Fonte: Os autores.

Relacionamos também a ocorrência quanto a publicações e estratos. Com relação ao estrato $A 1$, verificamos que três revistas apresentam uma ocorrência cada uma. Dos três artigos, a dimensão relacional (Carvalho, 2008) e a educação inclusiva (Oliveira, \& Leite, 2011) apresentaram-se distantes das referências procuradas nesse levantamento. Ao contrário, Guzzo e cols. (2010) apresentam um panorama sobre a consolidação da Psicologia Escolar e Educacional no Brasil, contribuindo para a definição dos aspectos teórico-instrumentais vinculados à categoria «b» - aquela que apresenta resultados de pesquisas sobre os fundamentos teóricos e filosóficos.

Constatamos também a ocorrência de 18 artigos no estrato A2,distribuídos em cinco periódicos. Estudos de Psicologia, da Pontifícia Universidade Católica de Campinas, traz sete textos, porém apenas o de Barbosa e Marinho-Araújo (2010) demonstrou relação direta com nossos objetivos. O caderno Psicologia, da Universidade de São Paulo, publicou cinco artigos contidos neste estudo, também com referências teórico-instrumentais relevantes. A revista $P$ sicologia: Ciência e Profissão veiculou três artigos selecionados, Psicologia em Estudo apresentou dois artigos e o periódico Psicologia: Teoria e Prática contribui com um trabalho.

Quanto aos estratos B1 e B2 importa salientar que são 37 artigos distribuídos em oito publicações - Psicologia Escolar e Educacional (Associação Brasileira de Psicologia Escolar e Educacional - ABRAPEE), Educação e Pesquisa (USP), Avaliação Psicológica (UFRGS), Revista Brasileira de Orientação Profissional (FFCLRP/USP), Revista Brasileira de Educação Especial (Associação Brasileira de Pesquisadores em Educação Especial - ABPEE), Revista de Psicologia Política(Sociedade Brasileira de Psicologia Política), Psicologia e Educação(PUC/SP) e Trabalhos em Linguística Aplicada (UNICAMP) -, sendo que a revista da ABRAPEE sozinha responde por 29 desses textos (aproximadamente $88 \%$ do total no estrato B1 e $49 \%$ do total geral do levantamento).

Outro aspecto analisado diz respeito ao conjunto dos autores com maior ocorrência. Identificar esses autores auxiliou no aprofundamento das pesquisas posteriores. Elaboramos uma planilha a partir dos nomes que figuravam na autoria dos artigos e quando o nome aparecia em mais de uma publicação o mesmo foi repetido no registro. Após elen- 
Tabela 2. Autores em função do número de artigos no corpus definitivo.

\begin{tabular}{lc}
\hline \multicolumn{1}{c}{ Autor } & Número de artigos \\
\hline MARINHO-ARAUJO, Claisy Maria & 7 \\
\hline GUZZO, Raquel Souza Lobo & 4 \\
\hline LIMA, Vanessa Aparecida Alves de. & 3 \\
\hline MEZZALIRA, Adinete Sousa da Costa & 3 \\
\hline MOREIRA, Ana Paula Gomes & 3 \\
\hline SOUZA, Marilene Proença Rebello de & 3 \\
\hline ANGELUCCI, Carla Biancha & 2 \\
\hline BARBOSA, Deborah Rosária & 2 \\
\hline CARVALHO, Tatiana Oliveira de & 2 \\
\hline FACCI, Marilda Gonçalves Dias & 2 \\
\hline RODRIGUES, Marisa Cosenza & 2 \\
\hline SILVA, Silvia Maria Cintra da & 2 \\
\hline SOUZA, Vera Lúcia Trevisan de & 2 \\
\hline TADA, Iracema Neno Cecilio & 2 \\
\hline BISINOTO, Cynthia ou OLIVEIRA, Cynthia Bisinoto de & 2 \\
\hline
\end{tabular}

Fonte: Elaboração dos autores.

Figura 2.

Distribuição das categorias por estrato

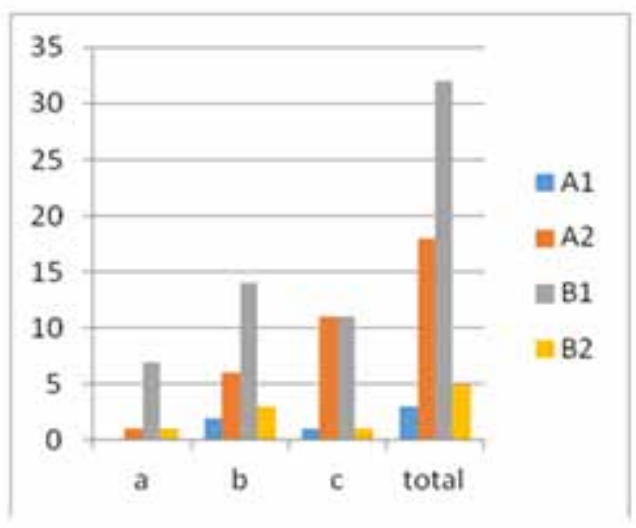

Fonte: elaboração dos autores
Distribuiçăo das categorias por ano

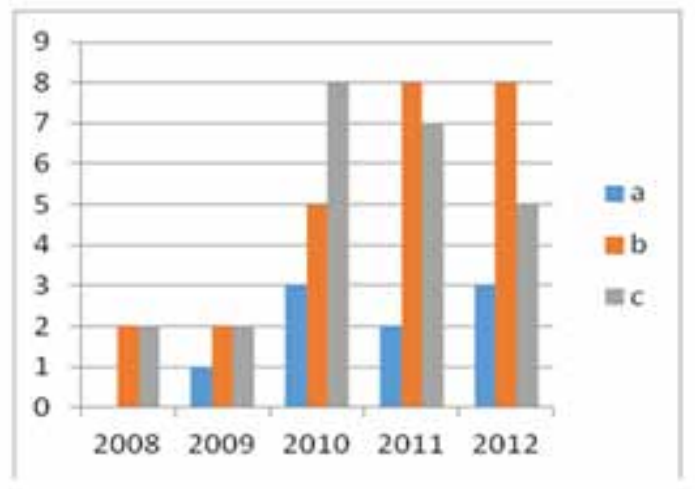

Fonte: elaboração dos autores carmos por ordem alfabética foram contadas as ocorrências, o que resultou na Tabela 2. No levantamento, o total de pesquisadores registrados é de 100, mas apenas aqueles mais significativos foram identificados aqui:

Assim, as informações sobre a ocorrência dos autores permitiram-nos reconhecer duas pesquisadoras que se destacam pela quantidade de trabalhos e pela relação com a pesquisa projetada - Claisy Maria Marinho-Araújo (UNB) e Raquel Souza Lobo Guzzo (PUCCAMP).

As ilustrações que produzimos a seguir relacionam: categorias $\mathrm{x}$ estratos do WebQualis e categorias $\mathrm{x}$ ano de publicação (Figura 2). O interesse era de identificar o impacto das publicações e a cronologia em que os textos foram publicados. A partir do que vimos acima, quanto aos estratos destaca-se o B1 e quanto ao ano de publicação prevalece o ano de 2011.

Nesta figura reconhecemos que as categorias "a" (fontes para estudos comparativos) e "b" (fundamentos teórico-metodológicos) - que compõem $58,6 \%$ do total desse levantamento - têm predomínio de artigos veiculados em revistas A2 (38,8\% do total nesse estrato) e B1 (65,6\% do total). Com relação ao período, demonstramos que a quanti- 
dade de textos publicados com alguma menção à Psicologia Escolar e Educacional - nos periódicos de maior fator de impacto em Psicologia e Educação - cresce consideravelmente e mantém-se estável entre os anos de 2010 de 2012. A título de exemplo, a categoria "b" que apresenta duas ocorrências em 2008 e em 2009 (totalizando 16\% do total nessa categoria), apresenta oito artigos em 2011 e em 2012 (atingindo 64\% nesse período).

\section{Discussão}

No que se refere à categorização, verificamos a distribuição dos artigos conforme os critérios da hipótese inicial: classificamos na categoria "a" os artigos de maior interesse para a pesquisa subsequente, num total de nove trabalhos. Esses são resultados que têm como objeto as ideias e as concepções que psicólogos apresentam sobre sua formação inicial (ou sobre sua atuação nas escolas) e como sujeitos psicólogos da área escolar e educacional, professores da educação ou alunos. Os textos classificados na categoria "b" tratam de aspectos teóricos e metodológicos que são subsídios para reflexões acerca da Psicologia nas escolas, contabilizando o maior número de artigos entre as categorias (total de 25). A categoria "c" reuniu os artigos com maior distância em relação aos objetivos desse levantamento, num total de 24 arquivos, relacionados a diversos referenciais e temas da Psicologia. Então, do total de 58 artigos registrados apenas os classificados nas categorias "a" e "b" (34 deles) foram escolhidos para serem analisados com maior profundidade.

O quadro de referências apresentou considerável número de trabalhos que tratam das teorias desenvolvidas e da formação profissional na Psicologia Escolar e Educacional do Brasil, ou relatam investigações empíricas que dialogam com o projeto a que este estudo está vinculado. $\mathrm{O}$ número de publicações na sondagem inicial (74 periódicos) sugere que muitos veículos divulgam vasta produção sobre Psicologia Escolar e Educacional no país. No entanto, dentro das categorias encontramos 58 artigos que foram encontrados em apenas 16 revistas - o que representa apenas $21,6 \%$ do total na sondagem.

Discutimos também outros aspectos, como por exemplo, o de que os estudos sobre a Psicologia Escolar e Educacional têm alcançado projeção na área de Psicologia, sendo que o maior número de artigos do corpus definitivo (58) foi encontrado no estrato B1 (32 artigos ou 55,2\%) enquanto no estrato B2, por exemplo, foram encontrados apenas cinco artigos (ou 8,6\%). Outra observação é a de que o número de publicações tem crescido recentemente. Com exceção do estrato $A 1$, no qual o número reduzido de ocorrências (3) interfere na qualidade da projeção, nos outros estratos a tendência é nítida. Nos textos das revistas classificadas como A2, a produção concentrou-se claramente nos últimos anos a partir de 2010 (88,9\% do total desse estrato), enquanto no estrato B1 o número cresce gradativamente até o último ano do levantamento - 6,25\% em 2008, 12,5\% em 2009, 25\% em 2010, estabilizando-se em 28,1\% em 2011 e 2012. Esse as- pecto pode indicar a tendência de crescimento quantitativo e qualitativo das pesquisas nesse campo de aplicação.

Dentre as revistas que mais contribuem para a investigação posterior, certamente a Psicologia Escolar e Educacional (ABRAPEE) é a mais importante com 29 textos selecionados. Inclusive, esse número representa $90,6 \%$ de todos os textos do estrato B1, enquanto outras quatro publicações contribuem com o restante de 9,4\%. Cumpre mencionar que embora o objetivo principal da ABRAPEE seja publicar trabalhos sobre Psicologia Escolar e Educacional em geral, grande parte desses textos não estava vinculada aos critérios eleitos aqui para as categorias "a" e "b".

Com relação aos autores, o primeiro destaque fica com a autora Claisy Maria Marinho-Araújo, que apresenta sete artigos neste levantamento. Além de apresentar um número significativo de trabalhos selecionados, a autora influenciava desde as leituras preliminares para o projeto. Aqui deixamos mais claro o papel dessa pesquisadora de cunho eminentemente teórico, na interface entre Psicologia e Educação. Por exemplo, o texto "Psicologia escolar no Brasil: considerações e reflexões históricas" (Barbosa, \& Marinho Araújo, 2010) influencia diretamente os fundamentos da pesquisa pretendida. Trata do panorama histórico sobre o contexto da produção acadêmica (teórico e institucional) no Brasil, o que define as condições históricas e materiais nas quais se realiza o trabalho do psicólogo escolar e educacional hoje. A leitura desse trabalho pode indicar tanto teorias e ideias para a justificativa metodológica, quanto possíveis representações que serão encontradas nos discursos dos sujeitos que relacionam psicologia e escola.

A segunda autora proeminente é Raquel Guzzo, que produziu quatro trabalhos reunidos neste levantamento. Em dois textos, encontramos estudos empíricos e bibliográficos que subsidiam a pesquisa ulterior. $O$ artigo "Psicólogo e escola: a compreensão de estudantes do ensino fundamental sobre esta relação" (Sant'Ana, Euzébios Filho, Junior, \& Guzzo, 2009) contém relatos dos sujeitos que sinalizam traços significativos importantes para a análise que pretendemos sobre os discursos de professores - como as ideias de que a Psicologia tem "respostas prontas" ou a de que alunos precisam mais de psicólogos do que outros atores no contexto escolar.

Já no trabalho "Psicólogo na rede pública de educação: embates dentro e fora da própria profissão" (Guzzo, Mezzalira, \& Moreira, 2012), a autora contribui para a reflexão sobre a inserção do psicólogo na escola pública. Apresenta como maiores empecilhos para a efetividade da proposta, de um psicólogo em cada unidade escolar pública, a ausência de formação e prática para atuação no espaço público (e na discussão sobre as políticas educacionais), bem como de práticas e de debate público sobre os contornos da própria formação em Psicologia Escolar e Educacional.

Outro aspecto curioso é que enquanto os periódicos são editados geralmente no Sudeste do Brasil, produções de outros estados foram encontradas com mais destaque no "corpus definitivo". Artigos importantes foram produzidos nesses contextos, como por exemplo: "Psicologia Escolar 
em Rondônia: formação e práticas" (Tada, Sápia, \& Lima, 2010), que trata da formação e atuação do psicólogo em escolas, por meio de questionários e diário de campo; "Psicologia Escolar na rede pública de educação dos municípios de Santa Catarina" (Tondin, Dedonatti, \& Bonamigo, 2010) e "A atuação do psicólogo no ensino público do Estado do Paraná" (Lessa, \& Facci, 2011).

A análise do material revelou contribuições fundamentais nos artigos selecionados dentro da categoria "a". Encontramos nove artigos, os quais discutimos a seguir.

No artigo classificado em A2, Ulup e Barbosa (2012) apresentam estudo sobre o estágio obrigatório desenvolvido na graduação em Psicologia da UFRJ - na disciplina Psicologia Escolar - com olhar para as representações que permeiam as relações na instituição concedente. Os autores defendem a postura crítica e emancipatória que veem desenvolvida nos graduandos, e asseveram que "cabe ao psicólogo auxiliar no resgate da autonomia do professor por meio de intervenções de potencialização e que o levem a investigar a dinâmica institucional" (p. 259). Esse referencial é importante por sinalizar uma imagem de Psicologia Escolar e Educacional mais abrangente, que supera o modelo clínico tradicional - "voltado para o diagnóstico e o tratamento de supostos distúrbios físicos e psíquicos situados no aluno" (Patto, 1997, p. 467).

O artigo classificado no extrato B2 tem autoria de Carvalho e Souza (2012) e trata de pesquisa realizada em três escolas particulares no município de Itabuna-BA. Os objetivos propostos e o método utilizado permitem aproximação com a nossa pesquisa, já que as Representações Sociais dos alunos no ensino fundamental (ciclo II) também abordam a Psicologia na escola. A maior relevância para nós refere-se ao paralelo que fazemos entre tema e campo de representação, já que sinaliza que o psicólogo escolar e educacional tem a função de promover "ajuda" ou "reduzir conflitos". Outra vez encontramos respaldo para consolidar alguns consensos sobre função e atuação do psicólogo em contexto escolar, como a indicação de que "cabe ao profissional de psicologia escolar empreender esforços no sentido de confirmar o seu campo de atuação de forma bem delimitada, a fim de ser reconhecido em sua função específica dentro da instituição escola" (Carvalho, \& Souza, 2012, p. 243).

Entre os artigos classificados como B1 mais um texto trata das representações dos alunos sobre a Psicologia na escola. Santıana, Euzebios Filho, Junior e Guzzo (2009) analisam desenhos e escrita de 240 alunos (ciclo I e II do ensino fundamental) da rede pública no Município de Campinas-SP. Com relação às representações, as categorias "Conversa sobre a vida" e "Ajuda quando existem problemas" estiveram presentes em $76 \%$ dos registros. Com relação aos pressupostos para atuação do psicólogo escolar e educacional, também concordam que devem estar pautados: na assessoria e na elaboração de programas de ensino; na melhoria das relações funcionais; no treinamento e desenvolvimento técnico-profissional; no apoio em questões relativas ao desenvolvimento humano; e em programas de prevenção à saúde.
Tada e cols. (2010) contribuem com artigo sobre a inserção do psicólogo na rede de ensino do estado de Rondônia. Por meio de procedimentos quantitativos e qualitativos, 38 psicólogos são acompanhados e analisados em suas representações. Os autores constatam o baixo impacto da formação continuada na atuação desses psicólogos. Fica patente que o público estrito desses profissionais seriam preferencialmente "pais e alunos" com $8 \%$ de ocorrência e "pais, alunos e professores" com 28\%. As observações sugerem que o foco da atuação se apresenta individualizado. Não obstante, os autores reforçam que a formação inicial, pioneira na região, aplica a abordagem "histórico-crítica" por meio de metodologia com forte cunho social.

Brasileiro e Souza (2010) apresentam estudo sobre a formação do psicólogo para a atuação sobre os processos de ensino-aprendizagem, também no estado de Rondônia. Realizam crítica ao modelo clínico, individualizante, e apresentam análise de questionários e entrevistas, com graduandos e agentes de ensino. Cumpre destacar a principal deficiência referida na formação inicial - "Relacionar teoria e prática dentro do currículo da formação do psicólogo" (33\% do universo analisado).

Tondin, Dedonatti e Bonamigo (2010) apresentam investigação realizada para retratar o panorama da legislação correspondente à atuação do psicólogo em escolas em 177 municípios do estado de Santa Catarina. As técnicas utilizadas foram entrevistas por telefone com secretários de educação municipais e análise documental sobre a legislação promulgada nos arquivos públicos municipais. Uma consideração importante é evocada quando afirmam que a atuação do psicólogo "poderia ser efetivada com maior rigor se fossem elaboradas diretrizes e parâmetros para os projetos de lei, pautadas pelas evoluções da Psicologia Escolar crítica, mediante a participação de profissionais de Psicologia e Educação" (Tondin, \& cols., 2010, p. 70).

As autoras Lessa e Facci (2011) apresentam resultado do trabalho sobre a atuação de psicólogos na rede de ensino do estado do Paraná. Com um universo de 385 psicólogos, distribuídos em 291 municípios que contam com a atuação do profissional nas escolas, a pesquisa retratou as representações desses psicólogos por meio de questionários. As categorias analisadas relacionavam-se a: público-alvo atendido (maioria destacando como "todos" na escola), modalidade de atuação (maioria atribuindo a "institucional" e "clínica"), projetos (concentrados na atuação junto aos professores) e autores que têm auxiliado na fundamentação teórica do trabalho (sendo que a maioria busca os recursos na área de Educação). Para os objetivos deste artigo, importa ressaltar que as autoras percebem a importância da Psicologia Escolar e Educacional também no nível institucional.

Souza, Ribeiro e Silva (2011) apresentam um trabaIho sobre a presença de psicólogos na rede particular de ensino em Uberlândia-MG. Os sujeitos compõem um conjunto de 12 profissionais e os procedimentos relacionaram-se à aplicação de questionários, bem como coleta de relatos, enquanto a análise dos dados registrou aspectos relacionados à formação e atuação deles. As constatações sugerem 
que a inserção do psicólogo escolar e educacional ainda é realizada de forma precária, tanto do ponto de vista conceitual quanto técnico, e identificam representações de caráter «instável e confuso» (p. 56)

Por fim, na categoria "a" encontramos o trabalho de Almeida (2012) que apresenta texto produzido a partir de uma "visão subjetiva". Como professora universitária, com extensa carreira na Educação, a pesquisadora desenvolve reflexão sobre a contribuição da Psicologia para a Educação. Cumpre destacar a afirmação que exemplifica, em nossa perspectiva, postura mais lúcida em relação ao papel da Psicologia no cotidiano das práticas escolares: "as correntes psicológicas são menos ambiciosas em relação a teorias globais e mais centradas em problemas. Poderia, assim, ajudar-me a ser melhor educadora e trabalhar para um ensino que promovesse a igualdade e a equidade de oportunidades para todos" (p. 347).

\section{Considerações finais}

Com o inventário tivemos o intuito de mapear, em base de periódicos específica, a produção acadêmica produzida sobre a função e a atuação do psicólogo nas escolas brasileiras. Dentre os artigos encontrados, foram mais relevantes para a continuidade dos estudos aqueles que relataram pesquisas empíricas de cunho qualitativo, nas áreas de Educação ou Psicologia Escolar e Educacional. Buscamos identificar informações que permitissem aprofundar o estudo das referências, assim como estabelecer com mais segurança os periódicos que contribuem com a maior quantidade de relatos de pesquisa. Esse procedimento foi importante para estabelecer a tendência de publicação nos estratos definindo o teor e a frequência, seguindo a cronologia, relacionando ano de edição e periódico em que foi publicado.

O marco teórico e alguns procedimentos específicos da investigação de mestrado mais ampla foram auxiliados pelos resultados demonstrados nesse levantamento - na medida em que sinalizam o estado atual das pesquisas na área, além da cronologia e da distribuição geográfica. Os autores mais recorrentes foram identificados, bem como as publicações com maior quantidade de contribuições, além de apresentar com destaque os resultados de pesquisas similares em outras unidades federativas afora Minas Gerais.

\section{Referências}

Almeida, L. R. (2012). Das relações entre educação e psicologia na perspectiva de uma educadora. Psicologia Escolar e Educacional, 16(2), 341-348.

Barbosa, D.R. (2012). Contribuições para a construção da historiografia da Psicologia educacional e escolar no Brasil. Psicologia, ciência e profissão, 32(spe), 104-123.
Barbosa, R. M., \& Marinho-Araujo, C. M. (2010). Psicologia escolar no Brasil: considerações e reflexões históricas. Estudos de Psicologia, 27(3) 393-402.

Brasileiro, T. S. A., \& Souza, M. P. R. (2010). Psicologia, diretrizes curriculares e processos educativos na Amazônia: um estudo da formação de psicólogos. Psicologia Escolar e Educacional (Impresso), 14(1). 105-120.

Brasil. Ministério do Trabalho e Emprego. (2010). Classificação Brasileira de Ocupações: CBO - 2010(3a ed.). Brasília: MTE, SPPE.

Carvalho, R. G. G. (2008). A dimensão relacional da intervenção dos serviços de psicologia nas escolas. Psicologia: Reflexão e Crítica, 21(1), 119-124.

Carvalho, I. S. C.; \& Souza, M. V. M. (2012) A representação social de alunos de escolas da rede particular de ensino acerca do papel do psicólogo escolar. Trab. linguist. apl. [online]., 51(1), 235-244.

Ferreira, N. S. A. (2002). As pesquisas denominadas "estado da arte". Educação \& Sociedade, 23(79).

Guzzo, R. S. L., Mezzalira, A. S., Moreira, A. P. G., Tizzei, R. P., \& Neto, W. M. D. F. S. (2010). Psicologia e educação no Brasil: uma visão da história e possibilidades nessa relação. Psicologia: teoria e pesquisa,26(25ANOS), 131-142.

Guzzo, R. S. L., Mezzalira, A. S. C., \& Moreira, A. P. G. (2012). Psicólogo na rede pública de educação: embates dentro e fora da própria profissão. Psicologia Escolar e Educacional, 16(2), 329338.

Lessa, P. V., \& Facci, M. G. D. (2011). A atuação do psicólogo no ensino público do Estado do Paraná. Psicologia Escolar e Educacional, 15(1), 131-141.

Martino, L. M. S. (2013). A disciplinarização da Epistemologia no ensino da(s) Teoria(s) da Comunicação, Intexto, 29, 1-17.

Mazzotti, T. B. (2006). Ciências da Educação em questão. Educação e Pesquisa, 32(3), 539-550.

Oliveira, M. A., \& Leite, L. P. (2011). Educação inclusiva: análise e intervenção em uma sala de recursos. Paidéia, 21(49), 197-205.

Patto, M. H. (1997). O papel social e a formação do psicólogo: contribuição para um debate necessário. Em M. H. Patto (Org.) Introdução à Psicologia Escolar (3a ed.). São Paulo: Casa do Psicólogo.

Sant'Ana, I. M., Euzébios Filho, A., Junior, F. L., \& Guzzo, R. S. L. (2009). Psicólogo e escola: a compreensão de estudantes do ensino fundamental sobre esta relação. Psicologia Escolar e Educacional, 13(1), 29-36. 
Souza, C. S., Ribeiro, M. J., \& Silva, S. M. C. (2011) A atuação do psicólogo escolar na rede particular de ensino. Psicologia Escolar e Educacional. (Impr.)[online]. 15(1), 53-61.

Tada, I. N. C., Sápia, I. P., \& Lima, V. A. A. (2010). Psicologia Escolar em Rondônia: formação e práticas. Psicologia Escolar e Educacional, 14(2), 333-340.
Tondin, C. F., Dedonatti, D., \& Bonamigo, I. S. (2010). Psicologia escolar na rede pública de educação dos municípios de Santa Catarina. Psicologia Escolar e Educacional, 14(1), 65-72.

Ulup, L., \& Barbosa, R. B. (2012). A formação profissional e a ressignificação do papel do Psicólogo no cenário escolar: uma proposta de atuação-de estagiários a psicólogos escolares. Psicol. Ciência e profissão, 32(1), 250-263.

Recebido em: 14/03/2014

Reformulado em: 08/06/15

Aprovado em: 10/06/2015

\section{Sobre os autores}

Erico Lopes Pinheiro de Paula (erico@proens.uftm.edu.br) Mestrando no Programa de Pós-graduação em Educação da Universidade Federal do Triângulo Mineiro - UFTM

Helena de Ornellas Sivieri-Pereira (helena.sivieri@gmail.com)

Professora adjunta no curso de Psicologia e professora efetiva do programa de pós-graduação em Educação da Universidade Federal do Triângulo Mineiro - UFTM

Este manuscrito foi produzido em 2013, no âmbito da disciplina obrigatória "Metodologia da Pesquisa" do PPGE/UFTM. Em sua versão preliminar foi apresentado como comunicação oral no evento "I International Conference on Teacher Education e IV Seminário de Formação de Professores da UFTM" com o título "O estado da arte sobre Psicologia Educacional/Escolar nas publicações do SciELO". 